Check for updates

Cite this: RSC Adv., 2018, 8, 5578

Received 13th December 2017

Accepted 29th January 2018

DOI: $10.1039 / c 7 r a 13304 a$

rsc.li/rsc-advances

\section{Room temperature amine sensors enabled by sidewall functionalization of single-walled carbon nanotubes $\uparrow$}

\author{
Clara Paoletti, ${ }^{a}$ Maggie He, ${ }^{\mathrm{b}}$ Pietro Salvo, (D) ac Bernardo Melai, ${ }^{a}$ Nicola Calisi, ${ }^{\text {ade }}$ \\ Matteo Mannini, (D) de Brunetto Cortigiani, ${ }^{\text {de }}$ Francesca G. Bellagambi, ${ }^{a}$ \\ Timothy M. Swager, ${ }^{\text {b }}$ Fabio Di Francesco*a and Andrea Pucci (D) *a
}

\begin{abstract}
A new series of sidewall modified single-walled carbon nanotubes (SWCNTs) with perfluorophenyl molecules bearing carboxylic acid or methyl ester moieties are herein reported. Pristine and functionalized SWCNTs ( $p$-SWCNTs and f-SWCNTs, respectively) were characterized by X-ray photoelectron spectroscopy (XPS), Raman spectroscopy and scanning electron microscopy (SEM). The nitrene-based functionalization provided intact SWCNTs with methyl 4-azido-2,3,5,6tetrafluorobenzoate (SWCNT-N- $\mathrm{C}_{6} \mathrm{~F}_{4} \mathrm{CO}_{2} \mathrm{CH}_{3}$ ) and 4-azido-2,3,5,6-tetrafluorobenzoic acid (SWCNT-N$\mathrm{C}_{6} \mathrm{~F}_{4} \mathrm{CO}_{2} \mathrm{H}$ ) attached every 213 and 109 carbon atoms, respectively. Notably, SWCNT-N- $\mathrm{C}_{6} \mathrm{~F}_{4} \mathrm{CO}_{2} \mathrm{H}$ was sensitive in terms of the percentage of conductance variation from 5 to $40 \mathrm{ppm}$ of ammonia $\left(\mathrm{NH}_{3}\right)$ and trimethylamine (TMA) with a two-fold higher variation of conductance compared to p-SWCNTs at $40 \mathrm{ppm}$. The sensors are highly sensitive to $\mathrm{NH}_{3}$ and TMA as they showed very low responses (0.1\%) toward $200 \mathrm{ppm}$ of volatile organic compounds (VOCs) containing various functional groups representative of different classes of analytes such as benzene, tetrahydrofurane (THF), hexane, ethyl acetate (AcOEt), ethanol, acetonitrile $\left(\mathrm{CH}_{3} \mathrm{CN}\right)$, acetone and chloroform $\left(\mathrm{CHCl}_{3}\right)$. Our system is a promising candidate for the realization of single-use chemiresistive sensors for the detection of threshold crossing by low concentrations of gaseous $\mathrm{NH}_{3}$ and TMA at room temperature.
\end{abstract}

\section{Introduction}

There has been an explosion of interest in carbon nanomaterials over the last 30 years. Carbon nanotubes (CNTs) have attracted a great deal of this attention because of their outstanding electrical, thermal, and mechanical properties as well as their high aspect ratio. ${ }^{1-4}$ Because of their delocalized electronic structure and the accessibility of their $\pi$-electronic states to external perturbations, the electrical properties of CNTs are also sensitive to changes in the local environment. ${ }^{5-9}$ This intrinsic property has been widely exploited to realize CNTbased sensors for different external stimuli, especially gases and

${ }^{a}$ Department of Chemistry and Industrial Chemistry, University of Pisa, Via G. Moruzzi 13, 56124 Pisa, Italy.E-mail: fabio.difrancesco@unipi.it; andrea.pucci@unipi.it ${ }^{b}$ Department of Chemistry, Institute for Soldier Nanotechnologies, Massachusetts Institute of Technology, 77 Massachusetts Avenue, Cambridge, MA 02139, USA 'Institute of Clinical Physiology, National Council of Research (IFC-CNR), Via G. Moruzzi 1, Pisa, 56124, Italy

${ }^{d}$ Department of Chemistry "U. Schiff”, University of Florence, Via della Lastruccia 3-13, 50019 Sesto Fiorentino (FI), Italy

${ }^{e}$ National Interuniversity Consortium of Materials Science and Technology (INSTM), Via G. Giusti 9, 50121 Firenze, Italy

$\dagger$ Electronic supplementary information (ESI) available. See DOI: 10.1039/c7ra13304a vapours. Moreover, the electrical nature of these responses allows for facile integration into other platforms such as the resonant circuits of a quartz crystal microbalance (QCM) or in a commercial RFID tag ${ }^{10}$ where a network analyser or a mobile phone ${ }^{5}$ can be used as the reader.

In particular, the detection of amines is important for industrial/environmental monitoring, ${ }^{11}$ food quality control ${ }^{12}$ and disease diagnosis..$^{13}$ For example, in food quality control, the levels of ammonia $\left(\mathrm{NH}_{3}\right)$, trimethylamine (TMA), dimethylamine and triethylamine can be used to assess the spoilage of fish. ${ }^{14}$ Devices capable of monitoring $\mathrm{NH}_{3}$ and TMA would enable quality validation throughout the food chain and identify those responsible for incorrect preservation. More specifically, fish is fresh if TMA remains below $10 \mathrm{ppm}$, whereas a concentration between $10 \mathrm{ppm}$ and $50 \mathrm{ppm}$ indicates preliminary rot, and above $60 \mathrm{ppm}$ fish is considered rotten. ${ }^{15}$

To date, several studies have been performed concerning the development of chemical sensors sensitive to $\mathrm{NH}_{3}$ and TMA. Most of them involved the use of metal oxides working at temperatures above $200{ }^{\circ} \mathrm{C} .{ }^{16,17}$ Recently, researchers have started combining the properties of metal-oxides and carbon nanomaterials, for example by decorating the surface of CNTs or graphene with metal-oxides nanoparticles. ${ }^{18}$ However, good results were only obtained at high temperatures and/or high 
concentrations of analyte. ${ }^{19}$ Notably, pristine carbon nanotubes (p-CNTs) change their conductivity upon the interaction with gas molecules, ${ }^{7}$ but the sensitivity and selectivity towards gas analytes are poor. Covalent or non-covalent modification of CNTs is an accessible procedure to provide graphitic materials with modulated functionalities and potential sensor response. ${ }^{6,20,21}$ Among the possible covalent functionalizations of CNTs, nitrene chemistry has proved to be an effective strategy under mild conditions being also useful for potential scale-up synthesis. ${ }^{22,23}$

In this work, we exploit the nitrene chemistry for the introduction on the SWCNTs surface of aziridinyl moieties that are able to provide an effective sensing response towards gaseous $\mathrm{NH}_{3}$ and TMA at room temperature. Notably, the degree of SWCNTs functionalization was determined by X-ray photoelectron spectroscopy (XPS), whereas Raman spectroscopy and scanning electron microscopy (SEM) assessed the structural integrity of CNTs after functionalization.

\section{Experimental}

\section{Chemicals and instrumentation}

SWCNTs were obtained from Nano-C Corp. (ultra-purified SWCNT, UPT200) and used without further purification. Methyl pentafluorobenzoate (99\% purity) was purchased from Sigma-Aldrich and used as received. Chromium (purity 99.99\%) and gold (purity 99.99\%) were purchased from R.D. Mathis. All chemicals were purchased from Sigma-Aldrich and used without further purification.

XPS analysis were performed as described by Salvo et al. ${ }^{24}$

${ }^{1} \mathrm{H}$ and ${ }^{19} \mathrm{~F}$ NMR data were recorded on a Bruker AVANCE III HD 400 instrument at $400 \mathrm{MHz}$ and $376 \mathrm{MHz}$, respectively. Chemical shifts are reported as $\delta$ values (ppm) and referenced to the residual protons of deuterated $\mathrm{CDCl}_{3}$. High resolution mass spectra were measured with a Bruker Daltonics APEXIV 4.7 Tesla FT-ICR-MS using ESI ionization.

Raman spectra were measured by a Horiba Jobin-Yvon LabRam (HR 800) Raman Confocal Microscope, with a laser excitation at $532 \mathrm{~nm}$ and a laser spot size of $1.2 \mu \mathrm{m}$. The Raman band peaks were calculated via Lorentzian curve fitting by the Levenberg-Marquardt algorithm.

Functionalized CNTs were characterized by SEM using a JEOL JSM-6700F field emission SEM (FESEM). CNTs analysis was performed using the public domain Image Tool 3.00 version image analyser program developed at the University of Texas Health Science Center in San Antonio and is available on Internet at http://ddsdx.uthscsa.edu/dig/itdesc.html.

An EmStat-MUX handheld potentiostat (PalmSens Instruments) was used to determine conductivity values from the sensor array.

A Fluke 287 True RMS (Fluke Corporation) was used as digital multimeter. Digital mass flow controllers (MFC) were from Sierra Instruments. A KINTEK gas generator system was used for gaseous VOCs detection measurements. Relative humidity was measured using a humidity meter (Extech).

The syntheses of methyl 4-azido-2,3,5,6-tetrafluorobenzoate (1) and of methyl 4-azido-2,3,5,6-tetrafluorobenzoic acid (2) were reported in the ESI. $\dagger$

\section{Preparation and characterization of functionalized SWCNTs}

As an example, an aliquot (20 mg) of SWCNTs was placed in a $100 \mathrm{~mL}$ Schlenk flask and dispersed in $20 \mathrm{~mL}$ of $N$-methyl-2pyrrolidone (NMP). The mixture was sonicated for $2 \mathrm{~h}$. The Schlenk flask was then equipped with a condenser and the suspension was bubbled with argon for $30 \mathrm{~min}$. An aliquot of $200 \mathrm{mg}$ of (1) was added to the mixture, which was then heated to $160{ }^{\circ} \mathrm{C}$ and left under argon atmosphere and constant stirring for $18 \mathrm{~h}$. The mixture was cooled at room temperature and the product was isolated by precipitation in acetone. The solid was recovered by centrifugation at $14000 \mathrm{rpm}$ for $20 \mathrm{~min}$. The separated solid was re-dispersed in $\mathrm{CHCl}_{3}$ with the aid of ultrasonication and then recovered by centrifugation at $13000 \mathrm{rpm}$ for $15 \mathrm{~min}$. The purification process was repeated four times. The final black solid (SWCNT-N-C $\mathrm{C}_{6} \mathrm{~F}_{4} \mathrm{CO}_{2} \mathrm{CH}_{3}$ ) was dried under vacuum at $70{ }^{\circ} \mathrm{C}$ overnight. An identical procedure was followed for the preparation of SWCNT-N-C ${ }_{6} \mathrm{~F}_{4} \mathrm{CO}_{2} \mathrm{H}$, by using 2.

Before the XPS analysis, some drops of analytical grade dichloromethane were added to dried samples. The solutions were sonicated for $5 \mathrm{~min}$ in an ultrasonic bath and the dispersion was immediately deposited on polycrystalline gold (about $100 \mathrm{~nm}$ thick) evaporated on mica. After the deposition, the samples were dried under nitrogen and annealed at $80{ }^{\circ} \mathrm{C}$ to remove the solvent and promote the sample adhesion to the substrate. XPS analysis was performed as detailed reported in the ESI. $\dagger$

\section{Fabrication of the electrodes array and sensitive films}

An aluminum mask was employed in the thermal evaporation (Angstrom Engineering) of 14 gold electrode arrays (1 mm gap) on microscope glass slides (VWR) that had been previously washed in acetone and dried. A $10 \mathrm{~nm}$ layer of chromium was deposited first to allow the subsequent adhesion of $100 \mathrm{~nm}$ of gold. A quantity of $2 \mathrm{mg}$ of pristine SWCNTs (p-SWCNTs) or functionalized carbon nanotubes (f-SWCNTs) was dispersed in $4 \mathrm{~mL}$ of $o$-DCB by sonication in an ultrasonic bath for 1-2 min at room temperature. The resulting dispersion was drop-cast onto the electrodes and dried under vacuum to remove the solvent. Typically, the deposition of 3-5 $\mu \mathrm{L}$ drops was necessary to obtain the target resistance of $100-150 \mathrm{k} \Omega$, checked by a digital multimeter. The 14-electrode array was used to test different materials under identical conditions.

\section{Delivering system of gases on the device}

The functionalized electrodes were placed in a flow chamber constructed from PTFE connected to a gas mixing and delivery system. This system consisted of two digital mass flow controllers to control the flow (0.5-4 $\mathrm{mL} \mathrm{min}^{-1}$ ) of $\mathrm{NH}_{3}$ or TMA in nitrogen $\left(1 \% \mathrm{NH}_{3}\right.$ in $\mathrm{N}_{2}$ and 1\% TMA in $\mathrm{N}_{2}$ custom-ordered from Airgas) and to dilute the target gas with $\mathrm{N}_{2}$ (0.5-1 $\left.\mathrm{L} \min ^{-1}\right)$ or air $\left(1 \mathrm{~L} \mathrm{~min}^{-1}\right)$.

The analytes were delivered to the device at various concentrations (5-40 ppm) for steps of $100 \mathrm{~s}$. For controlled humidity measurements, the gas mixture was bubbled through 
water before reaching the PTFE enclosure containing the device. The gas generator system was calibrated for each VOC of interest and used to deliver a known concentration of a given VOC diluted in $\mathrm{N}_{2}$ at a fixed gas flow rate to the device's enclosure. Relative humidity was also measured.

\section{Measurements of device response}

The conductivity values from the sensor array were determined by amperometric measurements. For this purpose, the current was measured with the PSTrace software (PalmSens BV) at constant voltage $(0.1 \mathrm{~V})$ between the electrodes. In this condition, the conductance ( $G=$ current/voltage) is directly proportional to current. To correct for differences between device resistances, the conductance is normalized such that $\Delta G / G_{0}=$ $\left(G_{0}-G\right) / G_{0}$, where $G_{0}$ is the conductance before exposure to $\mathrm{NH}_{3}$ or TMA and $G$ is the conductance achieved during exposure. In our work, the conductance decreased with analyte exposure and $\Delta G / G_{0}$ was positive. We report the responses as the arithmetic mean of the three replicated sensors for each material.

\section{Results and discussion}

\section{SWCNTs functionalization and characterization}

The azido group served as precursor to generate a highly reactive nitrene intermediate. Herein, the aryl nitrenes were formed by thermal treatment at $160{ }^{\circ} \mathrm{C}$ of methyl 4-azido-2,3,5,6tetrafluorobenzoate (1) and 4-azido-2,3,5,6-tetrafluorobenzoic acid (2) in NMP. This treatment allowed carboxylic acid and methyl ester moieties to be inserted on SWCNTs sidewalls (Fig. 1 and Table 1). Pentafluorophenyl compounds were used since their presence was supposed to foster the interaction of the sensitive material with the target analytes.

XPS analysis. The degree of functionalization of the modified SWCNTs was evaluated by XPS analysis (see ESI $\dagger$ ). The comparison of survey spectra confirmed that in the p-SWCNTs sample only carbon and oxygen are present (excluding the signals due to the gold substrate), whereas nitrogen and fluorine were detected in functionalized samples (Table 2). In good agreement with previous reports, ${ }^{25}$ the $\mathrm{C} 1 \mathrm{~s}$ region is characterized by 6 components: $\mathrm{C}=\mathrm{C}(284.53-284.38 \mathrm{eV}), \mathrm{C}-\mathrm{C}(285.5-$ $285.11 \mathrm{eV}), \mathrm{C}-\mathrm{O} / \mathrm{C}-\mathrm{N}(287.53-286.21 \mathrm{eV}$, our experimental setup

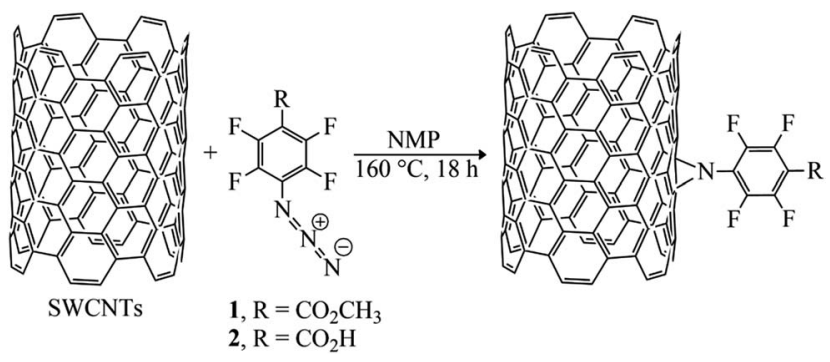

Fig. 1 Schematic reaction of SWCNTs with methyl-4-azido-2,3,5,6tetrafluorobenzoate (1) and 4-azido-2,3,5,6-tetrafluorobenzoic acid (2) via nitrene addition. did not allow these two components to be discriminated), $\mathrm{C}=\mathrm{O}$ (287.92-286.45 eV), $\mathrm{O}-\mathrm{C}=\mathrm{O}(289.54-288.39 \mathrm{eV}$ ), and $\pi-\pi$ (this component can be attributed to the delocalization of the electron on the surface of the nanotube). The $\mathrm{C}-\mathrm{F}$ contribution was not found because of the interference with the $\pi-\pi$ peak. Absorbed $\mathrm{CO}$ and $\mathrm{CO}_{2}$ within the porous structure of p-SWCNTs tubes possibly contributed to the carbonyl structure of the carbon peak. ${ }^{26}$

Fig. 2 shows the relative percentages of each component. After the functionalization, the $\mathrm{C}=\mathrm{C}$ component dramatically decreased and the $\mathrm{C}-\mathrm{C}$ and oxygenated components increased. The COO component was higher in the SWCNT-N- $\mathrm{C}_{6} \mathrm{~F}_{4} \mathrm{CO}_{2} \mathrm{CH}_{3}$ than in the SWCNT-N-C $\mathrm{F}_{4} \mathrm{CO}_{2} \mathrm{H}$. This difference can be attributed to the higher functionalization ratio of SWCNT-N$\mathrm{C}_{6} \mathrm{~F}_{4} \mathrm{CO}_{2} \mathrm{CH}_{3}$, which was also confirmed from the area of fluorine peak.

Table 2 shows that in p-SWCNTs the percentages of $\mathrm{F} 1 \mathrm{~s}$ and $\mathrm{N}$ 1s were $0 \%$, whereas there was a very low percentage of oxygen $(4.3 \%)$. In p-SWCNTs, the ratio between the component $\mathrm{C}=\mathrm{C}$ and $\mathrm{C}-\mathrm{C}$ was about 4.5 . These data confirmed that the oxidation of the p-SWCNTs sample was very low with a high unsaturation degree. In SWCNT-N-C ${ }_{6} \mathrm{~F}_{4} \mathrm{CO}_{2} \mathrm{CH}_{3}$ and SWCNT-N$\mathrm{C}_{6} \mathrm{~F}_{4} \mathrm{CO}_{2} \mathrm{H}$, the percentages of $\mathrm{F} 1 \mathrm{~s}$ were $1.7 \%$ and $0.9 \%$, respectively, whereas the ratio between the components $\mathrm{C}=\mathrm{C}$ and $\mathrm{C}-\mathrm{C}$ decreased to about 2 . In f-SWCNTs, the oxygen peak was higher than in p-SWCNTs. This result allowed the components $\mathrm{O}=\mathrm{C}$ and $\mathrm{O}-\mathrm{C}$ to be discriminated. Notably, the $\mathrm{O}=\mathrm{C}$ component had an equal percentage of the component $\mathrm{C}=\mathrm{O}$, which confirmed the fitting goodness. The fitting goodness could not be determined for the $\mathrm{O}-\mathrm{C}$ component, which could not be distinguished from the $\mathrm{C}-\mathrm{N}$ component because of a similar binding energy. ${ }^{25}$

The $\mathrm{N}$ 1s percentage was high when compared with the $\mathrm{F} 1 \mathrm{~s}$ percentage (every functional group had 1 atom of nitrogen and 4 atoms of fluorine). This result can be explained by a nitrogen contamination probably due to the residue of the solvent used in the functionalization reaction ( $N$-methyl-2-pyrrolidone: $\mathrm{NC}_{5} \mathrm{O}$ ). This residue affected not only the nitrogen area but also the carbon area. Therefore, using the area of $\mathrm{F} 1 \mathrm{~s}$ to calculate the expected area of nitrogen associated with the functional groups, we corrected the carbon area to eliminate the solvent contribution. The functionalization ratio for the SWCNT-N-C $\mathrm{C}_{6} \mathrm{~F}_{4} \mathrm{CO}_{2} \mathrm{H}$ sample was 1 functional group every 213 carbon atoms of SWCNTs and in the SWCNT-N-C $\mathrm{F}_{4} \mathrm{CO}_{2} \mathrm{CH}_{3}$ is 1 functional group every 109 carbon atoms of SWCNTs.

Raman spectroscopy. In Raman spectroscopy, the G-band (at about $1590 \mathrm{~cm}^{-1}$ ) is associated with the ordered $\mathrm{sp}^{2}$ hybridized carbon structure typical of graphite, whereas the D-band (at about $1330 \mathrm{~cm}^{-1}$ ) is due to the amorphous carbon and local defects that originate from structural imperfections. ${ }^{27}$ The general trend shows that after CNTs covalent functionalization, the G/D ratio decreases and the width of the D-band increases. ${ }^{28}$ Fig. 3 shows the Raman spectra for p-SWCNTs and f-SWCNTs and in Table 3 the modes and intensity peaks of D and $G$ bands are reported. Notably, because of the functionalization process, the intensity ratio of the $\mathrm{D}$ - and G-bands $\left(I_{\mathrm{D}} / I_{\mathrm{G}}\right)$ dropped over $50 \%$ whereas the full width at half maximum (FWHM) 
Table 1 Chemical structure and name of the sensitive compounds synthetised by nitrene chemistry

Name

increased importantly. This contradictory phenomenon can be addressed to the opposite effects of the removal of impurities and the creation of defects because of the functionalization. ${ }^{\mathbf{2 6}}$ Moreover, the opposite trend of the $I_{\mathrm{D}} / I_{\mathrm{G}}$ ratio can be also supported considering a recently published $[1,2]$ cycloaddition of CNTs by means of electron-poor aromatic nitrenes. ${ }^{29}$ In that work, the authors demonstrated that after the cycloaddition step, subsequent rehybridization restores the $\mathrm{sp}^{2}$ state, thus

Table 2 Components in p-SWCNTS and f-SWCNTs as determined by XPS analysis

\begin{tabular}{|c|c|c|c|c|c|c|}
\hline Sample & Component & Peak position (eV) & FWHM $(\mathrm{eV})$ & Sensitivity & Corrected area & Percentage \\
\hline \multirow[t]{7}{*}{ Pristine SWCNTs } & $F$ & - & - & 1 & 0 & $0.0 \%$ \\
\hline & $O$ & 531.8 & 3.1 & 0.7 & 408 & $4.3 \%$ \\
\hline & $\mathrm{C}=\mathrm{C}$ & 284.5 & 1.1 & 0.3 & 5963 & $63.2 \%$ \\
\hline & $\mathrm{C}-\mathrm{C}$ & 285.5 & 1.1 & 0.3 & 1297 & $13.7 \%$ \\
\hline & $\mathrm{C}-\mathrm{O} / \mathrm{C}-\mathrm{N}$ & 286.4 & 1.1 & 0.3 & 645 & $6.8 \%$ \\
\hline & $\pi-\pi$ & 290.5 & 1.1 & 0.3 & 338 & $3.6 \%$ \\
\hline & $C$ total & - & - & 0.3 & 9034 & $95.7 \%$ \\
\hline \multirow[t]{8}{*}{ SWCNTs- $\mathrm{CO}_{2} \mathrm{CH}_{3}$} & $F$ & 687.8 & 2.2 & 1 & 336 & $1.7 \%$ \\
\hline & $\mathrm{O}=\mathrm{C}$ & 531.6 & 1.9 & 0.7 & 1514 & $7.7 \%$ \\
\hline & $\mathrm{O}-\mathrm{C}$ & 533.2 & 1.9 & 0.7 & 523 & $2.7 \%$ \\
\hline & O total & - & - & 0.7 & 2037 & $10.3 \%$ \\
\hline & $\mathrm{C}=\mathrm{O}$ & 287.3 & 1.1 & 0.3 & 1503 & $7.6 \%$ \\
\hline & $\mathrm{COO}$ & 288.4 & 1.1 & $\mathrm{v}$ & 1179 & $6.0 \%$ \\
\hline & $\pi-\pi$ & 289.8 & 1.1 & 0.3 & 598 & $3.0 \%$ \\
\hline & $C$ total & - & - & 0.3 & 16034 & $81.3 \%$ \\
\hline \multirow[t]{8}{*}{ SWCNTs- $\mathrm{CO}_{2} \mathrm{H}$} & $F$ & 687.9 & 2.0 & 1 & 231 & $0.9 \%$ \\
\hline & $\mathrm{O}=\mathrm{C}$ & 531.6 & 2.1 & 0.7 & 1941 & $7.8 \%$ \\
\hline & $\mathrm{O}-\mathrm{C}$ & 533.5 & 2.1 & 0.7 & 650 & $2.6 \%$ \\
\hline & O total & - & - & 0.7 & 2591 & $10.4 \%$ \\
\hline & $N$ & 400.3 & 1.9 & 0.5 & 1618 & $6.5 \%$ \\
\hline & $\mathrm{C}=\mathrm{C}$ & 284.5 & 1.1 & 0.3 & 8936 & $35.8 \%$ \\
\hline & $\mathrm{C}-\mathrm{C}$ & 285.3 & 1.1 & 0.3 & 4236 & $17.0 \%$ \\
\hline & $\mathrm{C}-\mathrm{O} / \mathrm{C}-\mathrm{N}$ & 286.3 & 1.1 & 0.3 & 3385 & $13.6 \%$ \\
\hline
\end{tabular}




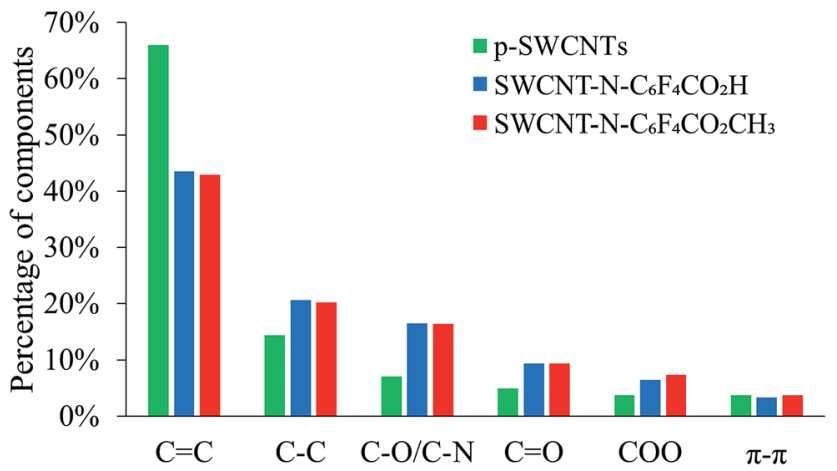

Fig. 2 Relative percentages of the components found in the $C 1 \mathrm{~s}$ region in $\mathrm{p}$-SWCNTs and f-SWCNTs after XPS analysis.

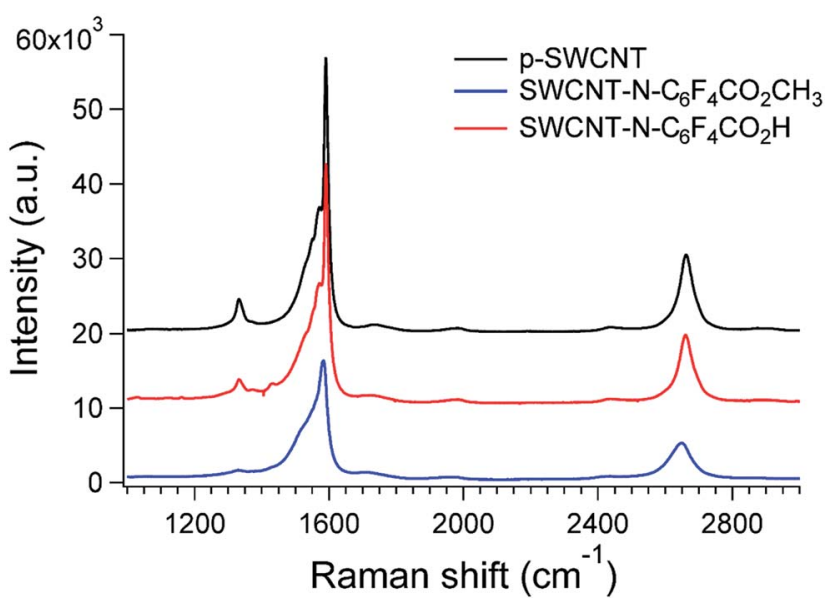

Fig. 3 Raman spectra (532 nm excitation) of $\mathrm{p}$ - and f-SWCNTs.

recovering the aromaticity of the system in the defected region of CNTs. Since this mechanism is found for electron deficient aromatic derivatives, a similar explanation can be also provided using azido-tetrafluorobenzoic derivatives as functionalizing agents.

SEM analysis. SEM analysis revealed the high density of wellexfoliated f-SWCNTs, which were not shortened or broken by the functionalization procedure (Fig. 4). This result confirms the effectiveness of the nitrene chemistry in providing undamaged functionalized CNTs thank to its mild reaction conditions. ${ }^{22,23}$

\section{Ammonia and trimethylamine gas sensing tests}

At room temperature, all sensors exhibited a maximum saturation response towards $\mathrm{NH}_{3}$ and TMA at $40 \mathrm{ppm}$. The response of SWCNTs after exposure to $40 \mathrm{ppm}$ of $\mathrm{NH}_{3}$ and TMA was assessed in $\mathrm{N}_{2}$ at controlled humidity conditions (three replicates per each of the CNTs). At relative humidity (RH) of $72 \%$, SWCNT-N- $\mathrm{C}_{6} \mathrm{~F}_{4} \mathrm{CO}_{2} \mathrm{H}$ was more responsive $\left(\Delta G / G_{0}(\%)=10.8 \pm\right.$ 0.7 and $9.3 \pm 0.8$, for $\mathrm{NH}_{3}$ and TMA respectively) than SWCNT$\mathrm{N}-\mathrm{C}_{6} \mathrm{~F}_{4} \mathrm{CO}_{2} \mathrm{CH}_{3}\left(\Delta G / G_{0}(\%)=4.7 \pm 0.2\right.$ and $5.5 \pm 0.8$, for $\mathrm{NH}_{3}$ and TMA respectively), whereas p-SWCNT had a negligible conductance variation (Fig. 5a). Our sensors also have good performance in air with similar variations, although with slightly lower responses (Fig. 5b).

The difference among f-SWCNTs is likely related to the strong Brønsted acid/base interaction of the carboxylic acid moiety with the lone-pair of the electron donor $\mathrm{NH}_{3}$ and TMA. Therefore, SWCNT-N- $\mathrm{C}_{6} \mathrm{~F}_{4} \mathrm{CO}_{2} \mathrm{H}$ was the best candidate for the realization of $\mathrm{NH}_{3} / \mathrm{TMA}$ gas sensors, thus it was subject to further investigation. Fig. $5 \mathrm{c}$ and d show the response of three replicate sensors exposed to 5,10 , and $40 \mathrm{ppm}$ of $\mathrm{NH}_{3}$ and TMA for $100 \mathrm{~s}$ each in air $(<5 \% \mathrm{RH})$, respectively ( 3 exposures for each concentration). The sensor response time was about $2 \mathrm{~min}$.

The decrease of conductance upon exposure can be explained considering the electronic nature of SWCNTs and their mechanisms of charge transfer. In the p-type semiconducting SWCNTs, the interaction with the donor molecules $\mathrm{NH}_{3}$ and TMA decreases the conductance of the network since the charge transfer from the amines effectively refills the holes in the valence band. ${ }^{30}$ This explains why p-SWCNTs are also sensitive to $\mathrm{NH}_{3}$ and TMA. The modification of the SWCNTs surface improves the interactions of the SWCNTs and enhances the electrical response.

Fig. 6a shows the calibration curves of SWCNT-N- $\mathrm{C}_{6} \mathrm{~F}_{4} \mathrm{CO}_{2} \mathrm{H}$ in response to $100 \mathrm{~s}$ exposure of $\mathrm{NH}_{3}$ and TMA over the range of 5-40 ppm. The limit of detection was found to be $0.2 \mathrm{ppm}$, a value that placed the designed device at the topmost positions in amine sensing based on SWCNTs. ${ }^{31}$ The enhanced sensitivity was ascribed to the functionalization process that also contributes in removal of loose SWCNTs agglomerates with poor electrical contact and a more efficient transport of carriers. Moreover, our design involved a more simple but effective functional probes with respect to those reported in the recent literature and based on combination of Au nanoparticles or functionalized polyanilines. ${ }^{31}$

A typical linear behaviour is gathered from semi-log plots. The higher sensitivity of the sensor towards the detection of TMA relative to $\mathrm{NH}_{3}$ can be ascribed to its more basic nature, indicating a more favoured lone electron pair interaction with SWCNTs and the pendant $\mathrm{N}_{-} \mathrm{C}_{6} \mathrm{~F}_{4} \mathrm{CO}_{2} \mathrm{H}$ groups. ${ }^{32}$ We observed a saturation above $40 \mathrm{ppm}$ (not shown) that was most likely due to a strong interaction between the gas molecules and SWCNT materials.

Table 3 Spectral features for D and G bands derived from Raman spectroscopy for $p$ - and f-SWCNTs

\begin{tabular}{llllr}
\hline Sample & D peak $\left(\mathrm{cm}^{-1}\right)$ & G peak $\left(\mathrm{cm}^{-1}\right)$ & $I_{\mathrm{D}} / I_{\mathrm{G}}$ & D band FWHM $\left(\mathrm{cm}^{-1}\right)$ \\
\hline p-SWCNTs & $1333.2 \pm 0.6$ & $1591.3 \pm 0.0$ & $0.12 \pm 0.01$ & $30.5 \pm 1.8$ \\
SWCNT-N-C $\mathrm{F}_{4} \mathrm{CO}_{2} \mathrm{H}$ & $1335.7 \pm 1.5$ & $1591.9 \pm 0.0$ & $0.06 \pm 0.0$ & $136.8 \pm 5.0$ \\
SWCNT-N-C ${ }_{6} \mathrm{~F}_{4} \mathrm{CO}_{2} \mathrm{CH}_{3}$ & $1328.2 \pm 1.6$ & $1584.2 \pm 0.2$ & $0.04 \pm 0.01$ & $56.8 \pm 9.0$
\end{tabular}



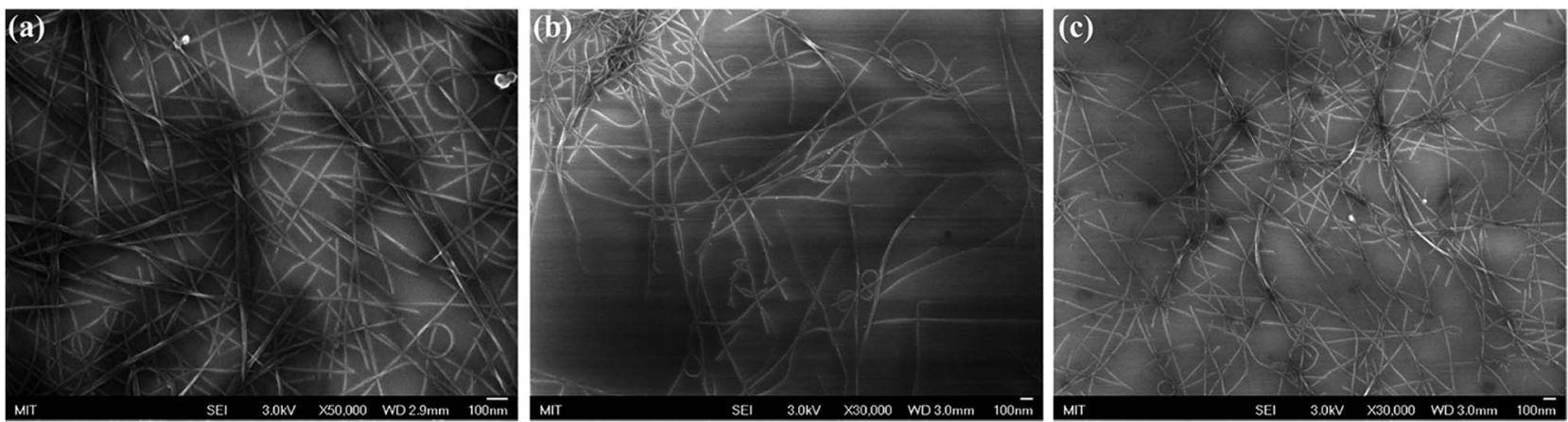

Fig. 4 Representative SEM images of (a) p-SWCNTs, (b) SWCNT-N- $\mathrm{C}_{6} \mathrm{~F}_{4} \mathrm{CO}_{2} \mathrm{CH}_{3}$ and (c) SWCNT-N- $\mathrm{C}_{6} \mathrm{~F}_{4} \mathrm{CO}_{2} \mathrm{H}$ samples. Typical accelerating voltages were $3.0 \mathrm{kV}$.
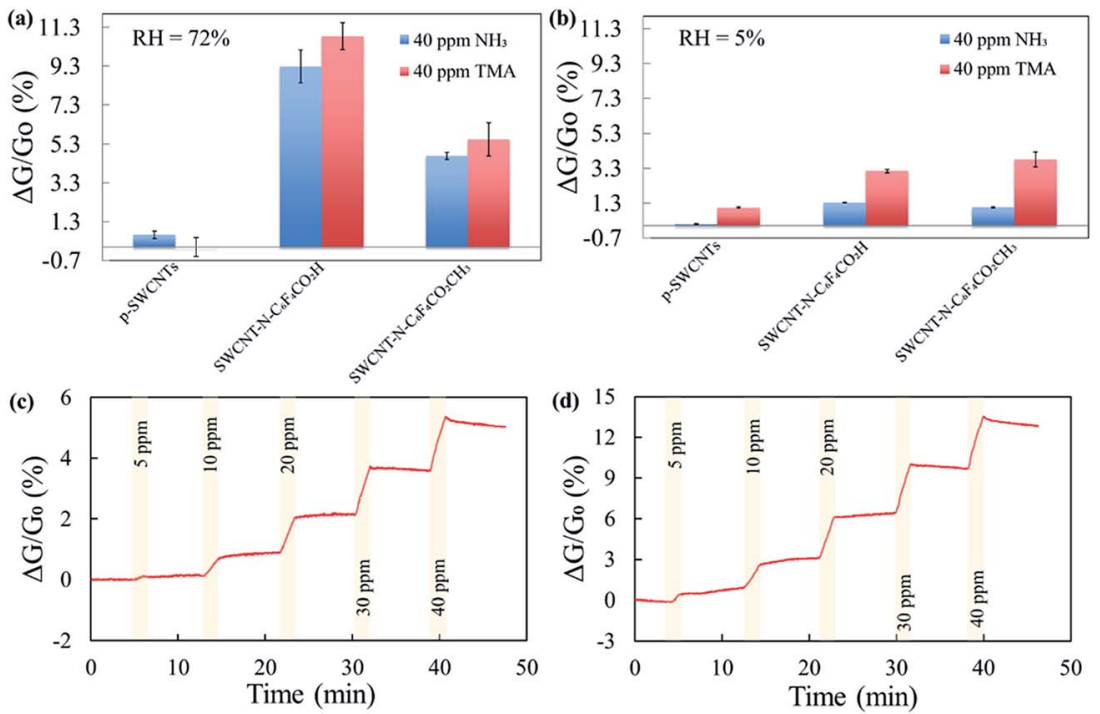

Fig. 5 Example of the responses of pristine and functionalized SWCNTs at different humidity (RH\%) conditions to exposures of 40 ppm of $\mathrm{NH}_{3}$ and TMA in (a) $\mathrm{N}_{2}$ at $\mathrm{RH}$ of $72 \%$ and (b) in air at $\mathrm{RH}<5 \%$; percentages of the conductance changes in $\mathrm{N}_{2}(<5 \% \mathrm{RH})$ of SWCNT-N-C $\mathrm{F}_{4} \mathrm{CO} \mathrm{O}_{2} \mathrm{H}$ in response to increasing concentrations of (c) $\mathrm{NH}_{3}$ and (d) TMA. The exposure time was $100 \mathrm{~s}$. A linear fit of the baseline was subtracted from the data.

We eventually checked the interference of other gases by observing the response of the sensor devices based on pristine and functionalized SWCNTs to a wide range of volatile organic compounds (VOCs) such as: benzene, tetrahydrofurane (THF), hexane, ethyl acetate (AcOEt), ethanol, acetonitrile $\left(\mathrm{CH}_{3} \mathrm{CN}\right)$, acetone and chloroform $\left(\mathrm{CHCl}_{3}\right)$. Very low interferences were observed from all the organic vapours investigated with $\Delta G / G_{0}$ (\%) values lower than 0.1 at $200 \mathrm{ppm}$ of VOCs (three replicates
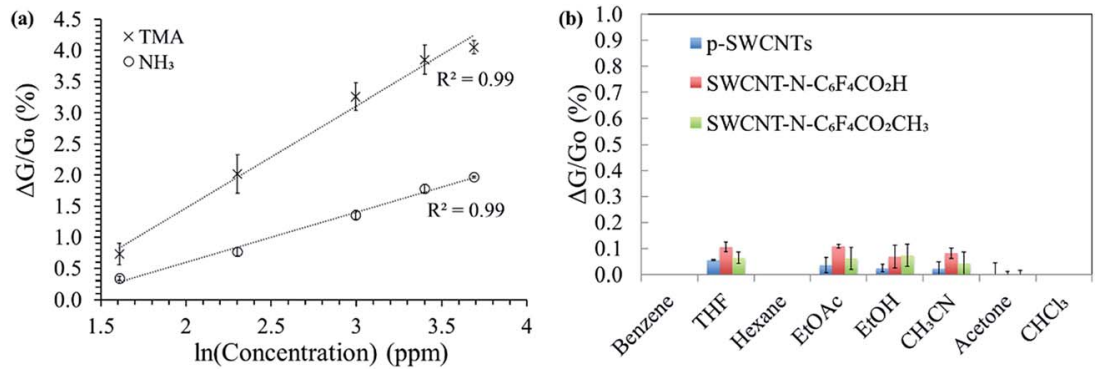

Fig. 6 (a) Response of SWCNT-N- $\mathrm{C}_{6} \mathrm{~F}_{4} \mathrm{CO}_{2} \mathrm{H}$ at a single exposure of various ppm concentrations (expressed as natural logarithm) of $\mathrm{NH}$ 3 and TMA in air. (Dotted line: linear fit $y=a+b \times x$. TMA: $a=-1.812 \pm 0.281$ and $b=1.641 \pm 0.097 ; \mathrm{NH}_{3}: a=-1.014 \pm 0.113$ and $b=0.807 \pm 0.039$ ); (b) dependence of the p-SWCNTs and f-SWCNTs from several VOCs. 
per each of the CNTs, Fig. 6b), indicating the high selectivity of the sensors toward $\mathrm{NH}_{3}$ and TMA.

When $\mathrm{NH}_{3}$ or TMA interacts with the perfluorinated functionalizing molecule, a change organic molecular component to an anion can also lead to depletion or trapping of the holes of CNTs to reduce the system conductance.

\section{Conclusions}

This work described the preparation of a chemiresistive sensor based on sidewall modified SWCNTs for the detection of low concentrations (5-40 ppm) of gaseous $\mathrm{NH}_{3}$ and TMA at room temperature. The XPS analysis confirmed the effectiveness of the nitrene-based functionalization in efforting 1 functional group every 213 and 109 carbon atoms for SWCNT-N-C $\mathrm{C}_{4} \mathrm{CO}_{2} \mathrm{H}$ and SWCNT-N-C $\mathrm{C}_{6} \mathrm{~F}_{4} \mathrm{CO}_{2} \mathrm{CH}_{3}$, respectively. Notably, f-SWCNTs were demonstrated to be at least 2 -fold more sensitive than $\mathrm{p}$ SWCNTs. It was worth noting that SWCNT-N-C $\mathrm{F}_{4} \mathrm{CO}_{2} \mathrm{H}$-based sensor was capable of discriminating the amount of $\mathrm{NH}_{3}$ and TMA emitted up to $40 \mathrm{ppm}$. Notably, the sensor remained responsive at high humidity, in the presence of air and showed no interference from all other gases of volatile organic compounds investigated, i.e. $\Delta G / G_{0}(\%)<0.1$ at $200 \mathrm{ppm}$ of VOCs. Efforts are being made to improve the output range and achieve a better discrimination between different volatile molecules. Nevertheless, this sensor is ready as a single-use indicator of the threshold crossing, about $10 \mathrm{ppm}$, of TMA and $\mathrm{NH}_{3}$ concentrations to monitor the freshness of packaged seafood products. Future combination with a RFID tag can lead to fast real-time seafood intelligent packaging.

\section{Conflicts of interest}

There are no conflicts to declare.

\section{Acknowledgements}

The financial support of the Italian Ministry of Education, Universities, and Research (MIUR) (PRIN 2010-2011 "Sensori chimici e tecniche strumentali accoppiate in spettrometria di massa per il controllo della sicurezza alimentare") and the University of Pisa (MIT-UNIPI Project "Functional Nanomaterials for the Detection of Volatile Amines (FUNDUS)") is gratefully acknowledged as is the National Science Foundation (DMR-1410718).

\section{Notes and references}

1 P. M. Ajayan, Chem. Rev., 1999, 99, 1787-1799.

2 R. H. Baughman, A. A. Zakhidov and W. A. de Heer, Science, 2002, 297, 787-792.

3 W. A. de Heer, MRS Bull., 2004, 29, 281-285.

4 J. M. Schnorr and T. M. Swager, Chem. Mater., 2011, 23, 646657.

5 J. M. Azzarelli, K. A. Mirica, J. B. Ravnsbæk and T. M. Swager, Proc. Natl. Acad. Sci. U. S. A., 2014, 111, 18162-18166.
6 Z. Ting, M. Syed, V. M. Nosang and A. D. Marc, Nanotechnology, 2008, 19, 332001.

7 L.-C. Wang, K.-T. Tang, I. J. Teng, C.-T. Kuo, C.-L. Ho, H.-W. Kuo, T.-H. Su, S.-R. Yang, G.-N. Shi and C.-P. Chang, Sensors, 2011, 11, 7763-7772.

8 N. Calisi, P. Salvo, B. Melai, C. Paoletti, A. Pucci and F. Di Francesco, Mater. Chem. Phys., 2017, 186, 456-461.

9 P. Salvo, N. Calisi, B. Melai, C. Paoletti, T. Lomonaco, A. Pucci, F. F. Di, V. Dini, M. Romanelli and A. Piaggesi, Int. J. Nanomed., 2017, 12, 949-954.

10 C. Occhiuzzi, A. Rida, G. Marrocco and M. Tentzeris, IEEE Trans. Microwave Theory Tech., 2011, 59, 2674-2684.

11 M. Chiesa, F. Rigoni, M. Paderno, P. Borghetti, G. Gagliotti, M. Bertoni, A. Ballarin Denti, L. Schiavina, A. Goldoni and L. Sangaletti, J. Environ. Monit., 2012, 14, 1565-1575.

12 S. F. Liu, A. R. Petty, G. T. Sazama and T. M. Swager, Angew. Chem., Int. Ed., 2015, 54, 6554-6557.

13 A. D. Wilson and M. Baietto, Sensors, 2011, 11, 1105-1176.

14 P. H. Wei, G. B. Li, S. Y. Zhao and L. R. Chen, J. Electrochem. Soc., 1999, 146, 3536-3537.

15 E.-X. Chen, H.-R. Fu, R. Lin, Y.-X. Tan and J. Zhang, ACS Appl. Mater. Interfaces, 2014, 6, 22871-22875.

16 B. T. Marquis and J. F. Vetelino, Sens. Actuators, B, 2001, 77, 100-110.

17 C. Sun and P. K. Dutta, Sens. Actuators, B, 2016, 226, 156-169. 18 S. Cui, S. Mao, Z. Wen, J. Chang, Y. Zhang and J. Chen, Analyst, 2013, 138, 2877-2882.

19 H. C. Su, M. Zhang, W. Bosze and N. V. Myung, J. Electrochem. Soc., 2014, 161, B283-B290.

20 N. Calisi, A. Giuliani, M. Alderighi, J. M. Schnorr, T. M. Swager, F. Di Francesco and A. Pucci, Eur. Polym. J., 2013, 49, 1471-1478.

21 F. Criscitiello, A. Scigliano, R. Bianco, M. R. Beccia, T. Biver and A. Pucci, Colloids Surf., A, 2017, 516, 32-38.

22 C. Gao, H. He, L. Zhou, X. Zheng and Y. Zhang, Chem. Mater., 2009, 21, 360-370.

23 M. Holzinger, O. Vostrowsky, A. Hirsch, F. Hennrich, M. Kappes, R. Weiss and F. Jellen, Angew. Chem., Int. Ed., 2001, 40, 4002-4005.

24 P. Salvo, N. Calisi, B. Melai, B. Cortigiani, M. Mannini, A. Caneschi, G. Lorenzetti, C. Paoletti, T. Lomonaco, A. Paolicchi, I. Scataglini, V. Dini, M. Romanelli, R. Fuoco and F. Di Francesco, Biosens. Bioelectron., 2017, 91, 870-877.

25 T. I. T. Okpalugo, P. Papakonstantinou, H. Murphy, J. McLaughlin and N. M. D. Brown, Carbon, 2005, 43, 153161.

26 E. D. Sosa, R. Allada, C. B. Huffman and S. Arepalli, XPS Protocol for the characterization of pristine and functionalized single wall carbon nanotubes, Nasa technical report, 2009.

27 K. A. Wepasnick, B. A. Smith, J. L. Bitter and D. Howard Fairbrother, Anal. Bioanal. Chem., 2010, 396, 1003-1014.

28 S. L. H. Rebelo, A. Guedes, M. E. Szefczyk, A. M. Pereira, J. P. Araujo and C. Freire, Phys. Chem. Chem. Phys., 2016, 18, 12784-12796.

29 A. Setaro, M. Adeli, M. Glaeske, D. Przyrembel, T. Bisswanger, G. Gordeev, F. Maschietto, A. Faghani, 
B. Paulus, M. Weinelt, R. Arenal, R. Haag and S. Reich, Nat. Commun., 2017, 8, 14281.

30 D. E. Johnston, M. F. Islam, A. G. Yodh and A. T. Johnson, Nat. Mater., 2005, 4, 589-592.
31 R. Tang, Y. Shi, Z. Hou and L. Wei, Sensors, 2017, 17, 882. 32 R. W. Friddle, M. C. Lemieux, G. Cicero, A. B. Artyukhin, V. V. Tsukruk, J. C. Grossman, G. Galli and A. Noy, Nat. Nanotechnol., 2007, 2, 692. 